\title{
Beam hardening: Analytical considerations of the effective attenuation coefficient of $\mathrm{x}$-ray tomography
}

\author{
J. Alles and R. F. Mudde ${ }^{a)}$ \\ Kramers Laboratorium voor Fysische Technology, Department of Multi-Scale Physics, \\ Delft University of Technology, Pr. Bernhardlaan 6, 2628 Delft, The Netherlands
}

(Received 12 December 2006; revised 16 April 2007; accepted for publication 24 April 2007; published 19 June 2007)

\begin{abstract}
Polychromatic $\mathrm{x}$-ray beams traveling though material are prone to beam hardening, i.e., the high energy part of the incident spectrum gets over represented when traveling farther into the material. This study discusses the concept of a mean attenuation coefficient in a formal way. The total energy fluence is one-to-one related to the traveled distance in case of a polychromatic beam moving through a given, inhomogeneous material. On the basis of this one-to-one relation, it is useful to define a mean attenuation coefficient and study its decrease with depth. Our results are based on a novel parametrization of the energy dependence of the attenuation coefficient that allows for closed form evaluation of certain spectral integrals. This approach underpins the ad hoc semianalytical expressions given in the literature. An analytical model for the average attenuation coefficient is proposed that uses a simple fit of the attenuation coefficient as a function of the photon energy as input. It is shown that a simple extension of this model gives a rather good description of beam hardening for x-rays traveling through water. (C) 2007 American Association of Physicists in Medi-
\end{abstract} cine. [DOI: 10.1118/1.2742501]

Key words: beam hardening, attenuation coefficient, effective energy, analytical model, spectra

\section{INTRODUCTION}

High-energy photons are used to penetrate into an object that is opaque. By studying the attenuation of the incident photon beams by the material, it is possible to reconstruct the internal structure of the object. This has many applications. For instance, in medical practice $\mathrm{x}$ rays are used for medical imaging. ${ }^{1}$ In the process industry $\gamma$ and $x$-ray photons are used to probe into steel vessels or opaque multiphase flows. ${ }^{2-4}$ For the latter, the temporal and spatial distribution of the different phases is an important parameter. In principle, a CT scanner can provide the required information.

However, in contrast to medical applications, the multiphase flow has small time scales, and the images need to be made at a relatively high rate, of the order of 100-200 frames per second. This requires rather strong sources, with serious consequences for safety. Moreover, the investments become high. These problems can be partially overcome by using $\mathrm{x}$-ray sources, such as the medical x-ray machines, instead of monoenergetic high-energy $\gamma$ sources. A disadvantage of these $\mathrm{x}$-ray beams is their broad energy spectrum. As a consequence, the elegant reconstruction using a single attenuation coefficient that depends only on the photon energy will no longer work properly. Instead, the low-energy end of the spectrum is attenuated most, a phenomenon called beam hardening. This prevents the easy use of a mean energy from which an effective attenuation coefficient can be derived.

However, the concept of an effective attenuation is appealing. Various attempts have been made to define this (see e.g., Refs. 5 and 6). Especially in the latter the author considered the effects of beam hardening in a formal way. Kleinschmidt quotes Bjärngard and Shackford, ${ }^{7}$ who stated that a clear and accepted definition of the attenuation of a broad spectrum of $\mathrm{x}$ rays is lacking. In his paper, Kleinschmidt considers this issue and provides a formal definition of the average attenuation coefficient, $\langle\mu\rangle$. In order to find a semianalytical expression for the attenuation coefficient as a function of the penetration depth $(x)$, several model functions were tested. These model functions were proposed ad hoc by Kleinschmidt and are chosen for their simplicity, i.e.,

$$
\begin{aligned}
& \langle\mu\rangle=\mu_{0}-\lambda x, \\
& \langle\mu\rangle=\frac{\mu_{0}}{1+\lambda x}, \\
& \langle\mu\rangle=\frac{\mu_{0}}{(1+\lambda x)^{2}}, \\
& \langle\mu\rangle=\mu\left(E_{\max }\right)+\frac{\mu_{1}}{1+\lambda_{1} x+\lambda_{2} x^{2}} .
\end{aligned}
$$

In this paper, we discuss a theoretical concept for the averaged attenuation coefficient and its behavior as a consequence of beam hardening. We address the error introduced and provide in Subsection III A an expansion in terms of the higher-order variances. Moreover, we present quantitative information as a function of the spectral width. We restrict the analysis to beam hardening in homogeneous media. The more general case of heterogeneous material is, obviously, much more complicated. Joseph and Ruth ${ }^{8}$ have discussed a method to correct beam hardening artifacts found in CT images attributable to bone and iodine by rescaling the attenuation of different substances to that of water. 
The paper is organized as follows. In Section II, we introduce the problem of beam hardening and introduce the relevant quantities such as fluence and attenuation. In Section III, we give the formal definition of the mean attenuation coefficient after passage of the $\mathrm{x}$ rays through a homogeneous material of thickness $x$. We will show that the error in the predicted fluence made by approximating the mean attenuation coefficient by its average over the incoming spectrum can be written as an infinite sum of higher-order variances. It follows that this approximation has an error of $\mathcal{O}\left(x^{2}\right)$. In Section IV, we will derive analytical expressions for the effective attenuation coefficient by approximating the attenuation coefficient $\mu(E)$. We will show that it is much more convenient to actually write this in the form $E=E(\mu)$ and use exponential functions as approximations for $E(\mu)$. Our method is based on a novel transformation of the integral over the photon energy into an integral over the attenuation coefficient itself. This transformation allows us to compute closed form solutions to various spectral integrals. Finally, in Section V, we compare the outcome of the analytical calculations to a completely numerical approach in which we discretize $\mu(E)$ and numerically solve the beamhardening problem.

\section{BEAM HARDENING}

\section{A. Monoenergetic radiation}

We will follow the formal definitions of Ref. 6 and start with the attenuation of a monoenergetic beam of photons with energy $E$. Consider a narrow, parallel beam of monoenergetic $\gamma$ or x rays incident on a unit area, $S$, of homogeneous material of thickness, $d x$. For an incident beam of intensity $\Phi$, the expected change in intensity after passing the material is given by

$$
d \Phi=-\mu(E) \Phi d x,
$$

where $\mu(E)$ is the energy-dependent linear attenuation coefficient. This quantity is a material property. The above equation leads to the well-known Lambert-Beer law for attenuation of high-energy photons:

$$
\frac{\Phi(x)}{\Phi_{0}}=e^{-\mu x} .
$$

\section{B. Polychromatic radiation}

In practice, $\mathrm{x}$-ray sources as used in medical applications produce photons with a wide energy spectrum, rather than monoenergetic ones. The photon spectrum can be characterized either by the spectrally distributed photon fluence, $\Phi_{E}$, or the spectrally distributed energy fluence, $\Psi_{E}=E \Phi_{E}$. Note that in case of a homogeneous material of thickness $d x$, also the Lambert-Beer law holds for $\Psi_{E}$ :

$$
d \Psi_{E}=-\mu(E) \Psi_{E} d x .
$$

The total photon fluence, $\Phi$, and the total energy fluence $\Psi$ are given by

$$
\begin{aligned}
& \Phi=\int \Phi_{E} d E, \\
& \Psi=\int \Psi_{E} d E=\int \Phi_{E} E d E .
\end{aligned}
$$

Since the attenuation coefficient is a function of $E, \mu$ $=\mu(E)$, the Lambert-Beer law no longer holds, and we have to replace this by its integral version: The photon intensity and the total energy fluence change when passing through a homogeneous piece of material of thickness $x$ as

$$
\begin{aligned}
& \Phi(x)=\int\left(\Phi_{E}\right)_{0} e^{-\mu(E) \cdot x} d E, \\
& \Psi(x)=\int\left(\Psi_{E}\right)_{0} e^{-\mu(E) \cdot x} d E .
\end{aligned}
$$

For most cases where $\mathrm{x}$ rays are used, $\mu(E)$ is a monotonically decreasing function of $E$. Consequently, low-energy photons are attenuated more than high-energy ones and the original photon-energy spectrum shifts toward the higher end of the original spectrum.

\section{Uniqueness}

In this subsection it will be shown that for a polychromatic beam, the mapping between the distance traveled through a given inhomogeneous medium of thickness $x$ and the radiant energy fluence rate $\Psi(x)$ is one-to-one.

For a narrow polychromatic beam of photons passing through a material of attenuation coefficient $\mu(E, x)$, the measured total energy fluence of the beam after traveling a distance $x$ is given by

$$
\Psi(x)_{\text {meas }}=\int\left(\Psi_{E}\right)_{0} e^{-\int_{0}^{x} \mu\left(E, x^{\prime}\right) d x^{\prime}} d E,
$$

with $\left(\Psi_{E}\right)_{0}$ the spectrally distributed energy fluence incident on the material. Note that any specific energy response of the detector has not been considered. Imagine now that we want to be able to uniquely determine $x$ from a measurement $\Psi_{\text {meas }}$. This will be possible only if the mapping between $x$ and $\Psi(x)_{\text {meas }}$ is one-to-one, or, in other words, if there are no two values of $x$ that lead to the same $\Psi(x)_{\text {meas. }}$. The properties of the mapping can be studied by considering the derivative of $\Psi(x)_{\text {meas }}$ with respect to $x$ :

$$
\begin{aligned}
\frac{d \Psi_{\text {meas }}}{d x} & =\frac{d}{d x} \int\left(\Psi_{E}\right)_{0} e^{-\int_{0}^{x} \mu\left(E, x^{\prime}\right) d x^{\prime}} d E \\
& =-\int\left(\Psi_{E}\right)_{0} \mu(E, x) e^{-\int_{0}^{x} \mu\left(E, x^{\prime}\right) d x^{\prime}} d E .
\end{aligned}
$$

Based on physical grounds it is obvious that $\left(\Psi_{E}\right)_{0}$ and $\mu(E, x)$ are all nonnegative functions. Hence the derivative will be negative for all values of $x$ no matter how $\mu$ is spatially distributed: 


$$
\frac{d \Psi_{\text {meas }}}{d x}<0, \quad \forall x .
$$

This proves that $\Psi(x)_{\text {meas }}$ is a monotonically decreasing function of $x$ and that the mapping of $\Psi_{\text {meas }}$ to $x$ is one-toone, for a given $\mu(x)$.

\section{AVERAGED ATTENUATION COEFFICIENT}

To deal with polychromatic beams an effective energy can be used. This is the energy of monochromatic photons that would have the same attenuation. In previous work, the existence of such an energy is postulated. ${ }^{6}$ Here we will give a mathematical treatment for the case in which the total photon energy is measured by the detectors (see also Ref. 1). Note that we assume perfect detectors to simplify the notation. Any response function of the detector, $\omega(E)$, can easily be incorporated in the equations. For clarity, we have set this response function equal to 1 , independent of the photon energy.

Consider a slab of material on which a polychromatic beam with energy distribution $\left(\Psi_{E}\right)_{0}$ is incident. After passing through a thickness $x$, the energy has changed from its initial distribution to $\Psi_{E}(x)$. Next, we consider the passage of the beam from $x$ to $x+d x$. Then the measured energy at $x+d x$ is

$$
\begin{aligned}
\Psi(x+d x)_{\text {meas }} & =\int \Psi_{E}(x) e^{-\mu(E) d x} d E \\
& \approx \int \Psi_{E}(x)[1-\mu(E) d x] d E \\
& =\Psi(x)_{\text {meas }}-\left[\int \Psi_{E}(x) \mu(E) d E\right] d x,
\end{aligned}
$$

with $\Psi(x)_{\text {meas }} \equiv \int \Psi_{E}(x) d E$. The second equality becomes exact for $d x \rightarrow 0$. Using $d \Psi(x)_{\text {meas }}=\Psi(x+d x)_{\text {meas }}-\Psi(x)_{\text {meas }}$ the above equation can be rewritten in the form of

$$
d \Psi(x)_{\text {meas }}=-\left[\frac{\int \Psi_{E}(x) \mu(E) d E}{\Psi(x)_{\text {meas }}}\right] \Psi(x)_{\text {meas }} d x .
$$

Upon comparing Eq. (16) with Eq. (7) we see that the quantity in brackets is the local measured attenuation coefficient, at position $x$. This local coefficient is identical to the attenuation coefficient averaged over the local photon spectrum:

$$
\mu_{\text {meas }} \equiv \frac{\int \Psi_{E}(x) \mu(E) d E}{\Psi(x)_{\text {meas }}}=\langle\mu\rangle_{\Psi(x)} .
$$

Solving Eq. (16) we can formally write

$$
\frac{\Psi(x)_{\text {meas }}}{\left(\Psi_{0}\right)_{\text {meas }}}=e^{-\int\langle\mu\rangle_{\Psi(x)} d x} .
$$

An effective energy can now be obtained by approximating the above integral for small $x$ :

$$
\int\langle\mu\rangle_{\Psi(x)} d x \approx\langle\mu\rangle_{\Psi_{0}} x .
$$

From Eq. (19) it is seen that the approximation becomes exact for vanishing $x$. Obviously, we now define the effective energy such that

$$
\mu\left(E_{\text {eff }}\right)=\langle\mu\rangle_{\Psi_{0}} .
$$

Equation (20) provides the effective energy, $E_{\text {eff }}$ based on the average of $\mu(E)$ with the incoming energy fluence, $\left(\Psi_{E}\right)_{0}$. Consequently, it provides the exact effective energy only in the case of vanishing thickness.

In many practical cases, the exact energy spectrum is not known, nor is the weighting characteristics of the detector. Then the effective energy is determined from experiments. ${ }^{9}$

With the effective energy, the measured energy can now be approximated as a monochromatic Lambert-Beer law:

$$
\Psi(x)_{\text {meas }} \approx \widetilde{\Psi}(x)_{\text {meas }}=\left(\Psi_{0}\right)_{\text {meas }} e^{-\langle\mu\rangle_{\Psi_{0} x}} .
$$

For large values of $x$ the estimate of the effective energy from Eq. (20) deteriorates, and the approximation becomes rather poor.

\section{A. Error analysis}

The difference between the true total energy fluence and the approximated one from Eq. (21) is

$$
\begin{aligned}
\mathcal{E} & =\text { Error }=\Psi(x)_{\text {meas }}-\tilde{\Psi}(x)_{\text {meas }} \\
& =\int\left(\Psi_{E}\right)_{0} e^{-\mu(E) x} d E-\left(\Psi_{0}\right)_{\text {meas }} e^{-\langle\mu\rangle_{\Psi_{0}} x} .
\end{aligned}
$$

Both terms on the right-hand side can be expanded in a Taylor series with respect to $x$, using $e^{-\alpha x}=1-(\alpha x / 1$ !) $+\left(\alpha^{2} x^{2} / 2 !\right)+\cdots$. After expanding the exponent $e^{-\mu(E) x}$ under the integral of the first term on the right-hand side of Eq. (22), we use that the integration runs over $E$, not over $x$. So, $x, x^{2}, \ldots$ are taken outside the integrals

$$
\begin{aligned}
\mathcal{E}= & \left(\Psi_{0}\right)_{\text {meas }}-\frac{x}{1 !} \int\left(\Psi_{E}\right)_{0} \mu(E) d E \\
& +\frac{x^{2}}{2 !} \int\left(\Psi_{E}\right)_{0} \mu^{2}(E) d E-\frac{x^{3}}{3 !} \int\left(\Psi_{E}\right)_{0} \mu^{3}(E) d E+\cdots \\
& -\left(\Psi_{0}\right)_{\text {meas }}\left(1-\frac{\langle\mu\rangle_{\Psi_{0}} x}{1 !}+\frac{\langle\mu\rangle_{\Psi_{0}}^{2} x^{2}}{2 !}-\frac{\langle\mu\rangle_{\Psi_{0}}^{2} x^{3}}{3 !}+\cdots\right) \\
= & \frac{x^{2}}{2 !}\left[\int\left(\Psi_{E}\right)_{0} \mu^{2}(E) d E-\frac{\left(\int\left(\Psi_{E}\right)_{0} \mu(E) d E\right)^{2}}{\int\left(\Psi_{E}\right)_{0} d E}\right] \\
& -\frac{x^{3}}{3 !}\left[\int\left(\Psi_{E}\right)_{0} \mu^{3}(E) d E-\frac{\left(\int\left(\Psi_{E}\right)_{0} \mu(E) d E\right)^{3}}{\left(\int\left(\Psi_{E}\right)_{0} d E\right)^{2}}\right]+\cdots .
\end{aligned}
$$

From the last equation, we see that the linear term in $x$ vanishes. Thus working with an effective attenuation coefficient based on the incoming spectrum is accurate up to order $x^{2}$. Further, we see that for a monochromatic beam, i.e., $\left(\Psi_{E}\right)_{0}=\delta(E)$ the delta function of Dirac, indeed we recover 
the Lambert-Beer law: The error is identical to zero. The same holds for the case when $\mu(E) \approx \mu$, i.e., when the attenuation is a very weak function of the energy in the range of the spectrum.

For simplicity we can rescale such that $\int\left(\Psi_{E}\right)_{0} d E=1$. Then the expression in the brackets of Eq. (23) can be interpreted as variances:

$$
\mathcal{E}=\sum_{n=2}^{\infty} \frac{(-x)^{n}}{n !}\left[\left\langle\mu^{n}\right\rangle_{\Psi_{0}}-\langle\mu\rangle_{\Psi_{0}}^{n}\right]
$$

with

$$
\left\langle\mu^{n}\right\rangle_{\Psi_{0}} \equiv \int\left(\Psi_{E}\right)_{0} \mu^{n}(E) d E
$$

and $\langle\mu\rangle_{\Psi_{0}}^{n} \equiv\left(\int\left(\Psi_{E}\right)_{0} \mu(E) d E\right)^{n}$

A relation between the magnitude of the beam hardening and the variance of the attenuation coefficient averaged over the spectrum has also been reported. ${ }^{5}$

\section{BEAM-HARDENING MODEL}

In this section a method will be presented that provides an analytical approximation for the function $\langle\mu\rangle_{\Psi(x)}$. We concentrate on a particular spectrum: a filtered Kramers spectrum. It will be shown, that an analytical expression for the effective attenuation coefficient can be derived for transmission through a slab of material of finite size $x$. For this, the attenuation coefficient, $\mu(E)$, needs to be known. We will investigate two possibilities. A first-order model, relating $\mu(E)$ as $\mu=c_{1}-c_{2} \ln E$ or in its inverse form, $E=A e^{-B \mu}$, and a second-order model, $E=A_{1} e^{-B_{1} \mu}+A_{2} e^{-B_{2} \mu}$. These implicit forms allow the construction of an analytical solution, which in the general case is not possible.

As initial spectrum $\left(\Psi_{E}\right)_{0}$ we take the Kramers spectrum, filtered by some material of thickness $L$ and attenuation coefficient $\mu(E)$ :

$$
\left(\Psi_{E}\right)_{0}=\frac{\left(E_{\max }-E\right)}{C} e^{-L \mu(E)},
$$

with $C$ a normalization factor with the same dimensions as $E$ (e.g., in $\mathrm{keV}$ ). For simplicity we have set $C$ equal to 1 . We also assume that the detectors have an ideal energy response.

It is convenient to shift the attenuation coefficient with respect to $\mu\left(E_{\max }\right)$ and define $\tilde{\mu}$ as

$$
\tilde{\mu}(E)=\mu(E)-\mu\left(E_{\max }\right) .
$$

We then rewrite the integral (12) (for the homogeneous case) as

$$
\begin{aligned}
\Psi(x)_{\text {meas }} & =\int_{0}^{E_{\max }}\left(\Psi_{E}\right)_{0} e^{-\mu(E) x} d E \\
& =g(x) e^{-L \mu\left(E_{\max }\right)} e^{-x \mu\left(E_{\max }\right)},
\end{aligned}
$$

with $g(x)$

$$
g(x)=\int_{0}^{E_{\max }}\left(E_{\max }-E\right) e^{-L\left[\mu(E)-\mu\left(E_{\max }\right)\right]} e^{-x\left[\mu(E)-\mu\left(E_{\max }\right)\right]} d E .
$$

An analytical integration of the above equation is for many functions $\mu(E)$ impossible. This is due to the $e^{\mu(E)}$ terms in the integral that runs over $E$. However, if we concentrate on $E(\mu)$ rather than $\mu(E)$ the integration over the energy $E$ can be easily transformed into an integral over $\tilde{\mu}$. This removes the problem of having to integrate $e^{\mu(E)}$ over $E$. The boundary values for $\tilde{\mu}$ are

1. $\tilde{\mu}(0)=\infty$,

2. $\tilde{\mu}\left(E_{\max }\right)=0$.

Thus we can write

$$
\begin{aligned}
g(x) & =\int_{0}^{E_{\max }}\left(E_{\max }-E\right) e^{-(L+x) \tilde{\mu}} d E \\
& =-\int_{0}^{\infty}\left(E_{\max }-E\right) e^{-(L+x) \tilde{\mu}}\left(\frac{d E}{d \tilde{\mu}}\right) d \tilde{\mu} .
\end{aligned}
$$

This integral is easily solved when $E(\mu)$ can be approximated by

$$
\cdot E(\mu) \approx \sum_{i=1}^{N} \alpha_{i} e^{\beta_{i} \tilde{\mu}}
$$

for $E(\mu)=\sum_{i=1}^{N} \alpha_{i} e^{\beta_{i} \widetilde{\mu}}$ boundary conditions 1 and 2 can be satisfied in a natural way by setting $\beta_{i}<0$.

If, alternatively, $E(\tilde{\mu})$ and hence $(d E / d \tilde{\mu})$ can be written in the form

$$
\cdot E(\mu) \approx \sum_{i=1}^{N} \alpha_{i} \tilde{\mu}^{\beta_{i}}
$$

the integral can be solved analytically via a Taylor series expansion of $e^{-x \tilde{\mu}}$ in terms of $x$ :

$$
\begin{aligned}
g(x) & =\sum_{n=0}^{\infty}(-1)^{n} \frac{x^{n}}{n !} \int_{0}^{E_{\max }}\left(E_{\max }-E\right) e^{-L \tilde{\mu}} \tilde{\mu}^{n} d E \\
& =-\sum_{n=0}^{\infty}(-1)^{n} \frac{x^{n}}{n !} \int_{0}^{E_{\max }}\left(E_{\max }-E\right) e^{-L \tilde{\mu}} \widetilde{\mu}^{n}\left(\frac{d E}{d \tilde{\mu}}\right) d \tilde{\mu} .
\end{aligned}
$$

The integral over $\tilde{\mu}$ is easy, as now the combination of an exponent and a power of $\tilde{\mu}$ renders the well-known gamma function: $\Gamma(z)=\int_{0}^{\infty} e^{-t} t^{z-1} d t$. In the next sections both a firstand second-order model for $E(\tilde{\mu})$ of the exponential form will be considered.

\section{A. First-order model for $E(\mu)$}

A simple attempt to describe $E(\tilde{\mu})$ that gives an easy integration of Eq. (29) is 


$$
E(\tilde{\mu})=A e^{-B \tilde{\mu}}
$$

From boundary value 2 it immediately follows that $A=E_{\max }$, such that only one free parameter remains. Substitution of this expression in Eq. (29) yields:

$$
g(x)=\frac{E_{\max }^{2} B}{B+L+x}-\frac{E_{\max }^{2} B}{2 B+L+x} .
$$

From Eq. (18) it follows that

$$
\langle\mu\rangle_{\Psi(x)}=\frac{d}{d x}\left[-\ln \Psi(x)_{\text {meas }}\right] .
$$

Combining Eq. (27) with the expression found for $g(x)$, we find

$$
\begin{aligned}
\langle\mu\rangle_{\Psi(x)} & =\frac{d}{d x}\left[-\ln \left(g(x) e^{-\mu\left(E_{\max } x\right)}\right)\right] \\
& =\mu\left(E_{\max }\right)+\frac{\eta_{1}}{1+\eta_{1} x}+\frac{\eta_{2}}{1+\eta_{2} x},
\end{aligned}
$$

with $\eta_{1}=(B+L)^{-1}$, and $\eta_{2}=(2 B+L)^{-1}$, both having units $\mathrm{cm}^{-1}$. Note that in the limit of $x \rightarrow \infty,\langle\mu\rangle_{\Psi(x)}$ reduces to $\mu\left(E_{\max }\right)$, which is physically correct.

\section{B. Second-order model for $E(\mu)$}

A further refinement, allowing a better fit of $\mu(E)$ to experimental data, can be achieved by using

$$
E(\tilde{\mu})=A_{1} e^{-B_{1} \tilde{\mu}}+A_{2} e^{-B_{2} \tilde{\mu}}
$$

The same procedure as for the first-order model has to be followed. We know from boundary value 2 that $E_{\max }=A_{1}$ $+A_{2}$. Hence the model has three free parameters. Substitution of the second-order expression into Eq. (29) we find for $g(x)$ :

$$
\begin{aligned}
g(x)= & -\frac{\left(A_{1}+A_{2}\right) A_{1} B_{1}}{B_{1}+L+x}-\frac{\left(A_{1}+A_{2}\right) A_{2} B_{2}}{B_{2}+L+x}+\frac{A_{1}^{2} B_{1}}{2 B_{1}+L+x} \\
& +\frac{A_{1} A_{2}\left(B_{1}+B_{2}\right)}{B_{1}+B_{2}+L+x}+\frac{A_{2}^{2} B_{2}}{2 B_{2}+L+x} .
\end{aligned}
$$

Finally, we calculated $\langle\mu\rangle_{\Psi(x)}$ :

$$
\begin{aligned}
\langle\mu\rangle_{\Psi(x)} & =\frac{d}{d x}\left[-\ln \left(g(x) e^{-\mu\left(E_{\max } x\right)}\right)\right] \\
& =\mu\left(E_{\max }\right)+\frac{\frac{\left(A_{1}+A_{2}\right) A_{1} B_{1}}{\left(B_{1}+L+x\right)^{2}}+\frac{\left(A_{1}+A_{2}\right) A_{2} B_{2}}{\left(B_{2}+L+x\right)^{2}}-\frac{A_{1}^{2} B_{1}}{\left(2 B_{1}+L+x\right)^{2}}-\frac{A_{1} A_{2}\left(B_{1}+B_{2}\right)}{\left(B_{1}+B_{2}+L+x\right)^{2}}-\frac{A_{2}^{2} B_{2}}{\left(2 B_{2}+L+x\right)^{2}}}{\frac{A_{1}+A_{1} B_{1}}{B_{1}+L+x}+\frac{\left(A_{1}+A_{2}\right) A_{2} B_{2}}{B_{2}+L+x}-\frac{A_{1}^{2} B_{1}}{2 B_{1}+L+x}-\frac{A_{1} A_{2}\left(B_{1}+B_{2}\right)}{B_{1}+B_{2}+L+x}-\frac{A_{2}^{2} B_{2}}{2 B_{2}+L+x}} .
\end{aligned}
$$

As can be seen, the structure of the above expression is rather simple: All terms in the numerator are the same as in the denominator except for the squaring of their individual denominators. This, however, makes it impossible to further reduce the expression. Nevertheless, once $\left\{A_{1}, A_{2}, B_{1}, B_{2}\right\}$ are known from fitting the second-order model to $E(\mu)$ calculating $\langle\mu\rangle_{\Psi(x)}$ is straightforward.

\section{DISCUSSION: BEAM HARDENING IN WATER}

To asses the quality of the derived beam-hardening models we consider a test case in which a Kramers spectrum filtered with $2.5 \mathrm{~mm}$ water propagates through water. Both beam-hardening models are compared with a numerical curve for the attenuation coefficient based on an accurate fit of the attenuation coefficient of water, using the expressions for the Compton (via the Klein-Nishina cross section) and photoelectric effect cross sections (see Ref. 10):

$$
\mu(E)=\zeta \mathrm{KN}(\alpha)+\frac{\xi}{E^{\tau}},
$$

with $\mathrm{KN}(\alpha)$ the Klein-Nishina cross section ${ }^{11}$ given by

$$
\begin{aligned}
\mathrm{KN}(\alpha)= & 2 \pi r_{e}^{2}\left\{\frac{1+\alpha}{\alpha^{2}}\left[\frac{2(1+\alpha)}{1+2 \alpha}-\frac{\ln (1+2 \alpha)}{\alpha}\right]\right. \\
& \left.+\frac{\ln (1+2 \alpha)}{2 \alpha}-\frac{1+3 \alpha}{(1+2 \alpha)^{2}}\right\}
\end{aligned}
$$

in which $\alpha=E / m_{0} c^{2}$, and $r_{e}=2.818 \times 10^{-15} \mathrm{~m}$ the classical electron radius. We have fitted this model to the Hubbell data for water. According to Ref. 10, the constant $\tau$ in Eq. (38) should be around 3. From the fit, we obtain for the constants: $\zeta=3.34 \times 10^{23} \mathrm{~cm}^{-3}, \quad \xi=6.19 \times 10^{-6} \mathrm{~cm}^{-1} \mathrm{MeV}^{2.96}$, and $\tau$ $=2.96$, respectively. In Fig. 1, the fitted analytical expression for $\mu(E)$ and the Hubbell data are shown for $E$ ranging from 20 to $150 \mathrm{keV}$. 


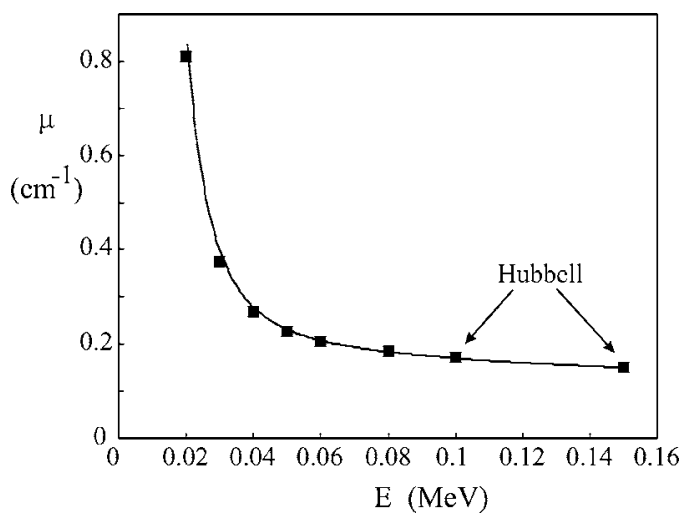

Fig. 1. Fit of the analytical model of Eq. (38) (solid curve) to the Hubbell table for water (

Next, we compute $\langle\mu\rangle_{\Psi(x)}$ using the above analytical expression for $\mu(E)$. This involves a number of steps given below.

1. We have computed $\mu(E)$ from Eq. (38) by discretizing $E$ from 1 to $150 \mathrm{keV}$ (step size $0.1 \mathrm{keV}$ ).

2. The Kramers spectrum after passing the water layer of $2.5 \mathrm{~mm}$ takes the form of Eq. (25), $\left(\Psi_{E}\right)_{0}=\left(E_{\max }\right.$ $-E) \exp (-\mu(E) \times L)$, with $L=2.5 \mathrm{~mm}$ water. It is computed with the values of $\mu(E)$ from step 1 .

3. Subsequently, the fluence $\Psi_{E}(x)$, at position $x$, is calculated $\Psi_{E}(x)=\left(\Psi_{E}\right)_{0} \exp (-\mu(E) x)$. Here, $x$ denotes the thickness of the water layer that the $\mathrm{x}$ rays are traveling through, $x$ varies from 0 to $25 \mathrm{~cm}$; it is discretized in steps of $\Delta=0.01 \mathrm{~cm}$.

4. Next, $\Psi(x)_{\text {meas }}=\int\left(\Psi_{E}\right)_{0} \exp (-\mu(E) x) d E$ is calculated using the trapezoidal method for the integral.

5. In the next step, $\int \Psi_{E}(x) \mu(E) d E$ is obtained using the trapezium rule.

6. Finally, we can compute $\langle\mu\rangle_{\Psi(x)}$ $=\int \Psi_{E}(x) \mu(E) d E / \Psi(x)_{\text {meas }}$ according to Eq. (17).

For the first- and second-order model, exponential fits for the water attenuation coefficient from the Hubbell tables ${ }^{12}$ have been made. Note that the Hubbell data closely follow the analytical expression for $\mu(E)$. We have used the Hubbell data for our first and second models to assess the capability of the models using a limited set of experimental data for the attenuation coefficient. The fitting range is from 20 to $150 \mathrm{keV}$. The fits are shown in Fig. 2. The coefficients are:

- First order: $A=E_{\max }=150 \mathrm{keV}, B=14.9 \mathrm{~cm}$.

- Second order: $A_{1}=109 \mathrm{keV}, B_{1}=29.184 \mathrm{~cm}, A_{2} \equiv E_{\max }$ $-A_{1}=41 \mathrm{keV}, B_{2}=1.11 \mathrm{~cm}$.

It is obvious, that the first-order fit is inadequate to describe the attenuation coefficient. The second-order fit, however, can follow the experimental data accurately. The results of the models and the direct calculation are shown in Fig. 3. The dashed-dotted curve in Fig. 3 corresponds to the numeri-

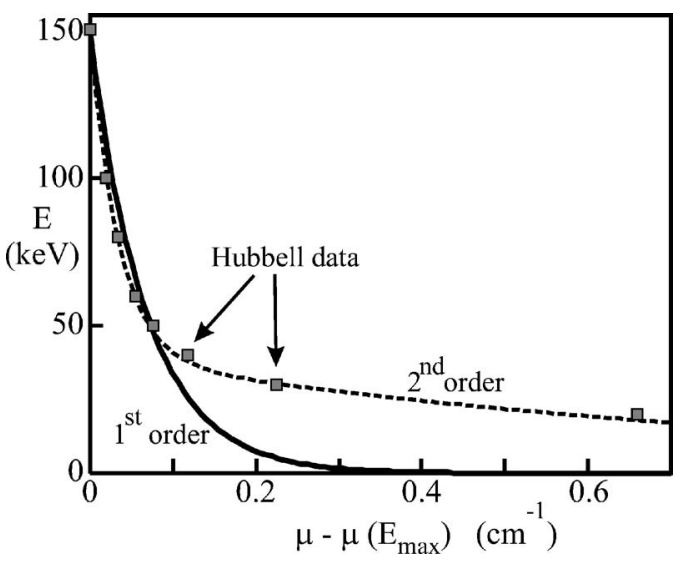

FIG. 2. Comparison of the first (solid curve) and second (dashed curve) order exponential fit of the attenuation coefficient as a function of photon energy to the Hubbell data for water.

cally obtained local attenuation coefficient $\langle\mu\rangle_{\Psi(x)}$. The second-order model follows the behavior of the real local attenuation coefficient quite accurately.

The parametrization proposed by Kleinschmidt [Eq. (4)]. $\langle\mu\rangle_{\Psi(x)}=\mu\left(E_{\max }\right)+\left(\mu_{1} / 1+\lambda_{1} x+\lambda_{2} x^{2}\right)$ that resembles our model closest can also be fitted to the numerical approach for $\langle\mu\rangle_{\Psi(x)}$. To keep Kleinschmidt's expression monotonic over the $x$ range we consider, the parameters $\left\{\lambda_{1}, \lambda_{2}\right\}$ are not fully free. We require the expression proposed by Kleinschmidt to have its minimum beyond the maximum thickness we investigate: $x_{\min }>25 \mathrm{~cm}$. The fit of Kleinschmidt's model to the numerical data is less accurate than our second-order model (see Fig. 4), but much better than the first-order model.

Our first-order model can be written in the form

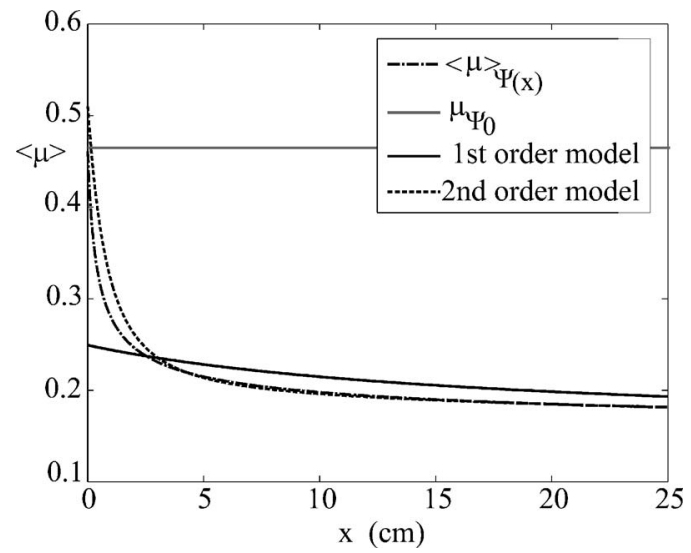

FIG. 3. Effective attenuation coefficient as a function of the thickness of the absorbing material. The dashed-dotted curve is the result of the numerical approach using Eq. (38). The horizontal, gray line represents the effective attenuation coefficient based on the incoming spectrum. The black solid curve is the result of the analytical beam hardening model using the firstorder exponential fit for $E(\mu)$, the dashed curve is for the second-order exponential fit. 

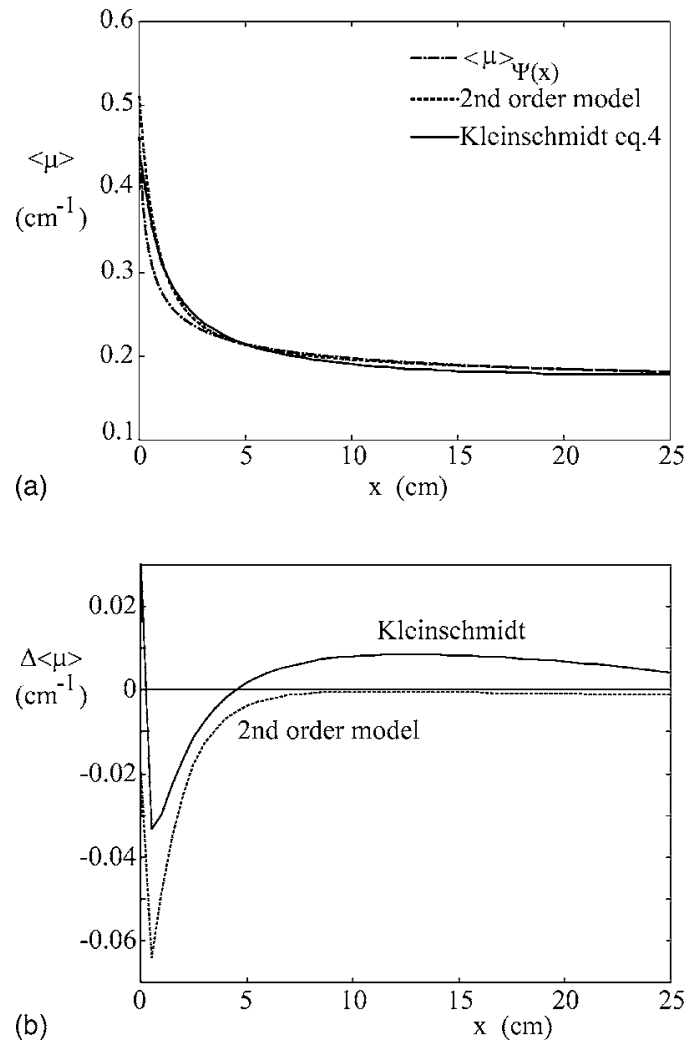

Fig. 4. Effective attenuation coefficient as a function of the thickness of the absorbing material. (a) Comparison of our numerical approach (dasheddotted curve) to the second-order model (dashed curve) and the relation proposed by Kleinschmidt (solid curve), $\langle\mu\rangle_{\Psi(x)}=\mu\left(E_{\max }\right)+\mu_{1} / 1+\lambda_{1} x$ $+\lambda_{2} x^{2}$. Fitting parameters: $\mu_{1}=0.2809 \mathrm{~cm}^{-1}, \quad \lambda_{1}=0.7882 \mathrm{~cm}^{-1}$, $\lambda_{2}=-0.0158 \mathrm{~cm}^{-1}$. (b) Difference $\Delta\langle\mu\rangle \equiv\langle\mu\rangle_{\text {num }}-\langle\mu\rangle_{\text {model }}$ of the secondorder model (dashed curve) and of Kleinschmidt's model (solid curve) with respect to the numerical approach.

$$
\langle\mu\rangle=\mu\left(E_{\max }\right)+\frac{\eta_{1}+\eta_{2}+2 \eta_{1} \eta_{2} x}{1+\left(\eta_{1}+\eta_{2}\right) x+\eta_{1} \eta_{2} x^{2}} .
$$

Hence Kleinschmidt's proposal coincides with our firstorder model only if either $\eta_{1}=0$ or $\eta_{2}=0$, which is not true. Reversely, Kleinschmidt's model can be rewritten as

$$
\begin{aligned}
\langle\mu\rangle & =\mu\left(E_{\max }\right)+\frac{\mu_{1}}{1+\lambda_{1} x+\lambda_{2} x^{2}} \\
& =\mu\left(E_{\max }\right)+\frac{\mu_{1}}{\lambda_{2}\left(x_{2}-x_{1}\right)}\left[\frac{1}{x-x_{1}}-\frac{1}{x-x_{2}}\right],
\end{aligned}
$$

with $\left\{x_{1}, x_{2}\right\}$ the two roots of $1+\lambda_{1} x+\lambda_{2} x^{2}=0$. Within the brackets, the two ratios have opposite signs. Again, this indicates that the ad hoc model of Kleinschmidt does not follow our first-order fit of $\mu(E)$.

Unfortunately, we did not manage to simplify the secondorder model [Eq. (37)], such that its structure is that of Kleinschmidt's proposal. Hence we cannot link the description of $\mu(E)$ that is underlying Kleinschmidt's fit to our suggestion. It is noted here that Kleinschmidt's original paper is concerned with high energy photons, whereas here the photon energy is up to $150 \mathrm{keV}$.
For the more realistic case where the filtering material is different from the material of interest, the thickness of the filtering material can be specified in an equivalent length of the material of interest and the entire procedure can be repeated.

\section{CONCLUDING REMARKS}

In this paper we have derived an analytical approximation of the effective attenuation coefficient that describes the attenuation of a beam of polychromatic $\mathrm{x}$ rays traveling through a homogeneous medium. The theoretical analysis is rigorous and takes into account the effects of beam hardening, that causes the high energy part of the incident $x$ rays to be over-represented after traveling a distance through the medium.

Second, we derived a beam-hardening model that describes the effective attenuation coefficient as a function of the distance traveled. The model can be used in a practical way if the attenuation coefficient is an invertible function of the energy. We specifically looked at the cases when the relation between the photon energy and the attenuation coefficient is of an exponential nature: $E=A e^{-B \mu}$, or $E=A_{1} e^{-B_{1} \mu}$ $+A_{2} e^{-B_{2} \mu}$. For these cases, the dependence of the mean attenuation coefficient on the material thickness $x$ can be analytically obtained. From the analysis it is clear that models for the mean attenuation proposed in the literature have an analytical base. For our first-order model, we have derived a simple relation between $\langle\mu\rangle_{\Psi}$ and the distance traveled, $x$, that resembles ad hoc models from the literature: $\langle\mu\rangle_{\Psi}$ $=\mu\left(E_{\max }\right)+\eta_{1} /\left(1+\eta_{1} x\right)+\eta_{2} /\left(1+\eta_{2} x\right)$. A simple recipe is given for the coefficients. A comparison of the first-order model with the exact one for water, shows that the model performs reasonably, but that there is room for improvement. With a second-order model $\left(E=A_{1} e^{-B_{1} \mu}+A_{2} e^{-B_{2} \mu}\right.$ with three fitting parameters), a much better agreement can be obtained. This model is capable of following quite closely the exact mean attenuation as a function of the distance traveled. Although the expression of the second-order model is rather lengthy, it relies on only three parameters and can easily be implemented into any computer code that analyzes the $\mathrm{x}$-ray data.

${ }^{\text {a) }}$ Author to whom correspondence should be addressed. Electronic mail: r.f.mudde@tudelft.nl

${ }^{1}$ E. C. McCullough, "Photon attenuation in computed tomography," Med. Phys. 2, 307-319 (1975).

${ }^{2}$ S. B. Kumar and M. P. Duduković, "Computer assisted gamma and X-ray tomography: Applications to multiphase flow systems," in Noninvasive Monitoring of Multiphase Flows, edited by J. Chaouki, F. Larachi, and M. P. Duduković (Elsevier, Amsterdam, 1997), Ch. 2, pp. 43-103.

${ }^{3}$ M. S. Beck, T. Dyakowski, and R. A. Williams, "Process tomography the state of the art," Trans. Inst. Meas. Control (London) 20, 163-177 (1998).

${ }^{4}$ S. B. Kumar, "Computed tomographic measurements of void fraction and modeling of the flow in bubble columns," Ph.D. thesis, Florida Atlantic University, 1994.

${ }^{5}$ C. Ruth and P. M. Joseph, "A comparison of beam-hardening artifacts in x-ray computerized tomography with gadolinium and iodine contrast agents," Med. Phys. 22, 1977-1982 (1995).

${ }^{6} \mathrm{C}$. Kleinschmidt, "Analytical considerations of beam hardening in medical accelerator photon spectrum," Med. Phys. 26, 1995-1999 (1999). 
${ }^{7}$ B. E. Bjärgard and H. Shackford, "Attenuation in high-energy x-ray beams," Med. Phys. 21, 1069-1073 (1994).

${ }^{8}$ P. M. Joseph and C. Ruth, "A method for simultaneous correction of spectrum hardening artifacts in ct images containing bone and iodine," Med. Phys. 24, 1629-1634 (1997).

${ }^{9}$ M. R. Millner, W. H. Payne, R. G. Waggener, W. D. McDavid, M. J. Dennis, and V. J. Sank, "Determination of effective energies in ct calibration," Med. Phys. 5, 543-545 (1978).
${ }^{10}$ F. H. Attix, Introduction to Radiological Physics and Radiation Dosimetry (Wiley, New York, 1986).

${ }^{11}$ D. Klein and Y. Nishina, "Uber die Streuung von Strahlung durch freie Elektronen nach der neuen relativistischen Quantumdynamik von Dirac," Z. Physik 52, 853 (1929).

${ }^{12}$ J. H. Hubbell and S. M. Seltzer, "Tables of X-ray mass attenuation coefficients and mass energy absorption coefficients," Nat. Inst. of Standards and Technology http://physics.nist.gov/PhysRefData (1996). 